Canadian

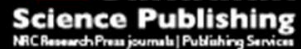

Canadian Journal of Physiology and Pharmacology Revue canadienne de physiologie et pharmacologie

\title{
Melatonin synergistically enhances protective effect of atorvastatin against gentamicin-induced nephrotoxicity in rat kidney
}

\begin{tabular}{|r|l|}
\hline Journal: & Canadian Journal of Physiology and Pharmacology \\
\hline Manuscript ID & cjpp-2015-0277.R1 \\
\hline Manuscript Type: & Article \\
\hline Date Submitted by the Author: & 06-Aug-2015 \\
\hline Complete List of Authors: & $\begin{array}{l}\text { Mehrzadi, Saeed; Iran University of Medical Sciences, } \\
\text { Kamrava, Seyed; Iran University of Medical Sciences } \\
\text { Dormanesh, Banafshe; AJA University of Medical Science } \\
\text { Motevalian, Manijeh; Razi Drug Research Center, Department of } \\
\text { Pharmacology, School of Medicine, Iran University of Medical Sciences } \\
\text { Hosseinzadeh, Azam; Iran University of Medical Sciences, } \\
\text { Hosseini Tabatabaei, Seyed; Shahid Beheshti University of Medical } \\
\text { Sciences, } \\
\text { Ghaznavi, Habib; Zahedan University of Medical Sciences, }\end{array}$ \\
\hline Keyword: & \begin{tabular}{l} 
melatonin, atorvastatin, gentamicin, nephrotoxicity, oxidative stress \\
\hline
\end{tabular} \\
\hline
\end{tabular}




\section{Melatonin synergistically enhances protective effect of atorvastatin against gentamicin-induced nephrotoxicity in rat kidney}

Saeed Mehrzadi ${ }^{1}$, Seyed Kamran Kamrava ${ }^{2}$, Banafshe Dormanesh ${ }^{3, *}$, Manijeh Motevalian ${ }^{1}$, Azam Hosseinzadeh ${ }^{1}$, Seyed Mohammad Taghi Hosseini Tabatabaei ${ }^{4}$, and Habib Ghaznavi ${ }^{5}$

${ }^{1}$ Razi Drug Research Center, Department of Pharmacology, School of Medicine, Iran University of Medical Sciences, Tehran, Iran

${ }^{2}$ ENT- Head \& Neck Research Center, Hazrate Rasoul Akram Hospital, Iran University of Medical Sciences, Tehran, Iran

${ }^{3}$ Department of Pediatric Nephrology, AJA University of Medical Science, Tehran, Iran

${ }^{4}$ Department of Pediatric Nephrology, Shahid Beheshti University of Medical Sciences, Tehran, Iran.

${ }^{5}$ Cellular and Molecular Research Center, Zahedan University of Medical Sciences, Zahedan, Iran

*Correspondence: Dr. Banafshe Dormanesh, Department of Pediatric Nephrology, AJA University of Medical Sciences, Tehran, Iran. Tel/Fax: 98-21-88622523.

E-mail: dr.dormanesh@yahoo.com 
Abstract: The risk of serious side-effects as nephrotoxicity is the principal limitation of gentamicin (GEN) therapeutic efficacy. Oxidative stress is considered to be an important mediator of GEN-induced nephrotoxicity. The present study was designed to evaluate the efficacy of the combination of melatonin (MT) plus atorvastatin (ATO) against GEN-induced nephrotoxicity in rats. We utilized 30 male Wistar albino rats allocated in five groups each containing six rats: control, GEN (100 mg/kg/day), ATO (10 mg/kg/day) + GEN, MT (20 mg/kg/day) + GEN and ATO (10 mg/kg/day) plus MT (20 mg/kg/day) + GEN. Kidneys weight, serum creatinine and urea concentration, renal ROS, MDA, GSH levels, SOD and CAT activity were determined. GEN-induced nephrotoxicity was evidenced by marked elevations in serum urea and creatinine, kidneys weight, renal ROS and MDA levels and reduction in renal GSH level, SOD and CAT activity. MT pretreatment significantly lowered the elevated serum creatinine concentration, kidneys weight, renal ROS and MDA levels but ATO couldn't reduce these parameters but similar to MT it was able to enhance the renal GSH level, CAT and SOD activity. In addition, combination therapy of MT plus ATO enhanced the beneficial effects of ATO while didn't change MT effects or even improve it. The present study indicates that combination therapy of MT plus ATO can attenuate renal injury in rats treated with GEN, possibly by reducing oxidative stress and it seems MT can enhance beneficial effects of ATO.

Key words: melatonin; atorvastatin; gentamicin; nephrotoxicity; oxidative stress; rats 


\section{Introduction}

Gentamicin (GEN), an aminoglycoside, was first isolated from Gram-positive bacteria, Micromonospora purpurea, and it has antibiotic activity against various gram-negative microorganisms and widely applied for treatment of life-threatening gram-negative infections (Balakumar et al. 2010). Because of its broad-spectrum activity, rapid bactericidal action, postantibiotic effects, chemical stability, low cost and its efficacy against germs insensitive to other antibiotics, it is a first-line drug in a variety of clinical situations in spite of the introduction of newer and less toxic antibiotics against Gram-negative microorganisms (Lopez-Novoa et al. 2011; Shakil et al. 2008). However, the risk of serious side-effects as ototoxicity and nephrotoxicity is the principal limitation of GEN therapeutic efficacy. Since kidneys play an importance role to maintain total fluid volume and acid base balance, nephrotoxicity is the major adverse effect of GEN that markedly limits its clinical use (Begg and Barclay 1995; Mathew 1992). The specificity of GEN nephrotoxicity may be related to its re-absorption and accumulation in renal proximal convoluted tubules, reaching a concentration of 5-50 times higher than plasma level in tubular renal cells, inducing lysosomal phospholipidosis process leading to the loss of tubular renal cells brush border integrity and necrosis (AbdelRaheem et al. 2009; Balakumar et al. 2010). GEN nephrotoxicity is a complex situation characterized by marked increased levels of serum creatinine, blood urea nitrogen (BUN) concentration, and significant decrease in glomerular filtration rate (GFR), extensive tubular epithelial cells vacuolization and desquamation, tubular fibrosis, epithelial edema, glomerular hypertrophy and severe proximal renal tubular necrosis, which eventually lead to renal failure and dysfunction (Dam et al. 2012; Romero et al. 2009; Soliman et al. 2007). 
Atorvastatin (ATO) belongs to statins group of cholesterol-lowering agents that effectively lowers serum cholesterol levels by competitively inhibiting 3-hydroxy3-methylglutaryl coenzyme A (HMG-CoA) reductase, the rate limiting enzyme of the mevalonate pathway, widely used in the primary and secondary cardiovascular diseases (Kapur and Musunuru 2008; Kwak et al. 2000). In addition to antihypercholesterolemic effects, statins have other significant effects such as antiinflammatory, antioxidant, immunomodulatory (Greenwood et al. 2006; Shishehbor et al. 2003; Yoshida 2003). Cholesterol-independent pleotropic effects of statins have led to suggestions that statins might be useful in management of several diseases. New evidences have shown statins reduce oxidative stress markers, nitrotyrosine and F2-isoprostane, as a result of inhibition of enzymatic source of oxygen free radicals such as NADPH oxidase (Davignon et al. 2004; Pignatelli et al. 2010; Wassmann et al. 2002).

Melatonin (MT, N-acetyl-5-methoxytryptamine) is the main secretory product of the pineal gland synthesized from tryptophan (Arendt, 1995). It has been shown other organs such as brain, eyes, gastrointestinal tract, skin, bone marrow, immune cells, some reproductive organs, and endocrine glands are also involved in production of MT (Reiter 1991; Sehajpal et al. 2014). MT is known to participate in many important physiological functions, including sexual behavior, sleep, immune function, and circadian rhythm (Ozler et al. 2011; Reiter et al. 2000; Reiter et al. 2005). MT also has beneficial roles in pathophysiological processes such as management of cardiovascular complication, migraine, cluster headache and convulsion therapy (Luchetti et al. 2010). Additionally, MT is a broadspectrum antioxidant and a free radical scavenger; not only it is able to remove reactive oxygen species (ROS) and reactive nitrogen species (RNS) by radical scavenger activity but also increases activity of antioxidant enzymes such as 
superoxide dismutase (SOD), catalase (CAT), glutathione reductase (GRd) glutathione peroxidase (GPX) and the expression of important endogenous antioxidant, glutathione (GSH) (Allegra et al. 2003; Rodriguez et al. 2004).

The investigation was carried out with the objective of evaluating the efficacy of MT plus ATO combination against GEN-induced nephrotoxicity in Wistar albino rats.

\section{Materials and methods}

\section{Drugs and chemicals}

Gentamicin (Sigma, USA), melatonin (Sigma, USA) and atorvastatin (Sigma, USA) were purchased from commercial sources.

\section{Test animals}

The experimental protocols used in this study were approved by the Ethics and Animal Care Committee of Zahedan University of Medical Sciences and were performed in accordance with the National Institute of Health Guidelines for the Care and Use of Laboratory Animals. Male Wistar rats with an initial body weight of 230-260 $\mathrm{g}$ were used in this study. The animals were kept in groups and housed in stainless steel cages under standard environmental conditions at a temperature of $23 \pm 3^{\circ} \mathrm{C}$, with $12 \mathrm{~h} \mathrm{light/dark} \mathrm{cycles.} \mathrm{Standard} \mathrm{commercial} \mathrm{diet} \mathrm{and} \mathrm{water} \mathrm{were}$ available ad libitum. The study was conducted in accordance with the European Communities Council Directive in such a way to minimize the number of animals and their suffering. 


\section{Experimental design}

30 male Wistar albino rats were randomly divided into five groups each containing six rats: group I, served as control group and consecutively was injected intraperitoneally (i.p.) with physiological saline $(1 \mathrm{ml} / \mathrm{kg} /$ day $)$ for 8 consecutive days. Group II, was administered with gentamicin sulfate $(100 \mathrm{mg} / \mathrm{kg} / \mathrm{day}$, i.p. $)$ for 8 consecutive days. Groups III and IV, were injected with MT (20 mg/kg/day, p.o.) (through a gavage) or ATO (10 mg/kg/day, p.o.) (through a gavage) for 7 consecutive days and then received injections of gentamicin sulfate (100 $\mathrm{mg} / \mathrm{kg} /$ day, i.p.) for 8 consecutive days. Group V, was administered a combination of MT (20 mg/kg/day, p.o.) and ATO (10 mg/kg/day, p.o.) for 7 consecutive days and then received injections of gentamicin sulfate $(100 \mathrm{mg} / \mathrm{kg} / \mathrm{day}$, i.p.) for 8 consecutive days.

\section{Serum and tissue collection}

After 8 days, the weight of animals was measured thereafter they were anesthetized by intraperitoneal injection of ketamine $(30 \mathrm{mg} / \mathrm{kg})$. Blood samples $(4-6 \mathrm{ml})$ were taken by cardiac puncture after which animals were sacrificed by cervical dislocation, kidneys quickly being removed, washed with ice-cooled physiological saline and absolute and relative (organ to body weight ratio) weights of kidney were measured for all rats. Kidneys were shock-frozen in liquid nitrogen and were kept in $-80^{\circ} \mathrm{C}$ for lipid peroxidation and ROS assay.

\section{Serum biochemical assays}


To obtain serum, blood samples were centrifuged at $1,500 \times g$ for $10 \mathrm{~min}$ at $+4^{\circ} \mathrm{C}$. Serum blood urea nitrogen (BUN) and creatinine levels were determined using an autoanalyzer (BT-TARGA-3000 model).

\section{Estimation of lipid peroxidation}

Malondialdehyde (MDA) concentrations were measured in kidney tissues as a marker of lipid peroxidation. Kidney tissues were homogenized in ice-cold tamponade containing $150 \mathrm{mM} \mathrm{KCl}$ for the determination of MDA. As previously described, MDA, referred as thiobarbituric acid reactive substance (TBARS), was determined by the absorbance of $535 \mathrm{~nm}$ wavelength and the results were expressed as malondialdehyde equivalents (nmol/mg protein) (Ohkawa et al. 1979).

\section{Assay of superoxide dismutase activity}

SOD activity was determined by using a commercially available SOD assay kit (Sigma) according to the manufacturer's instructions.

\section{Dichlorofluorescein assay for determination of ROS levels}

ROS levels in kidney were determined by using 2', 7'dichlorofluoresceindiacetate (DCF-DA) fluorescence method as described (Shinomol 2007). The fluorescence intensity of DCF was measured by a fluorescence plate reader using excitation at $485 \mathrm{~nm}$ and emission detection at $528 \mathrm{~nm}$ and results were expressed as fluorescence change percentage, where the control group was taken as $100 \%$.

\section{Determination of GSH level}

Renal GSH level was determined according to previous method (Kuo et al. 1983) with little modifications. The method is based on the reduction of 5,5-dithiobis-2- 
nitrobenzoicacid by GSH to yield a yellow component. The absorbance of this yellow component was measured at $412 \mathrm{~nm}$ and compared with the standard curve to determine GSH concentration.

\section{Determination of CAT activity}

CAT activity was determined by the method of Aebi (1984) using $\mathrm{H} 2 \mathrm{O} 2$ as substrate. A decrease in absorbance due to $\mathrm{H} 2 \mathrm{O} 2$ degradation was monitored spectrophotometrically at $240 \mathrm{~nm}$ for $1 \mathrm{~min}$ and the enzyme activity was expressed as $\mu \mathrm{mol} \mathrm{H} 2 \mathrm{O} 2$ consumed $/ \mathrm{min} / \mathrm{mg}$ protein.

\section{Statistical analysis}

The results were reported as mean \pm S.E.M. The statistical analyses were performed using one way analysis of variance (ANOVA) by SPSS software (v.20). Group differences were calculated by post hoc analysis using Tukey test.

\section{Results}

\section{Treatment effects of ATO, MT and a combination of them on kidney and body weight in GEN-treated rats}

No deaths or remarkable signs of external toxicity were observed in groups of animals that were given GEN, MT, ATO or a combination of MT and ATO. Relative kidney weight significantly increased $(p<0.01)$ in the GEN treated group compared with control group which could be ameliorated significantly by MT and a combination of MT and ATO pretreatment $(p<0.05)$ whereas ATO pretreatment didn't show any effect on relative kidney weight in comparison with GEN treated group. Body weight was not affected in any animal groups (Table1). 
Effects of ATO, MT and a combination of them on serum creatinine and urea levels in GEN-treated rats

In control animals, serum creatinine and urea concentration were $0.71 \pm 0.06$ and $23.4 \pm 1.1 \mathrm{mg} \mathrm{dl}^{-1}$ respectively. They significantly increased $(P<0.01$ and $P<$ 0.001 , respectively) in GEN treated group. Pretreatment with MT for 7 consecutive days significantly prevented from GEN-induced increase in serum creatinine and urea levels $(p<0.05$ and $p<0.01$, respectively). ATO pretreatment wasn't able to prevent GEN-induced increase in serum urea and creatinine levels. In MT and ATO combination group, serum creatinine and urea concentration were $0.72 \pm 0.03$ and $26.4 \pm 1.13 \mathrm{mg} \mathrm{dl}^{-1}$ respectively that were significantly lower than GEN ( $p<$ 0.01 and $\mathrm{p}<0.001$, respectively) and ATO $(p<0.05)$ treated groups (Table2).

\section{Effects of ATO, MT and a combination of them on renal ROS and MDA levels in GEN-treated rats}

MDA and ROS levels were elevated highly and significantly in the kidneys of GEN-intoxicated rats in comparison with control group $(P<0.001)$. Pretreatment of rats with MT significantly decreased renal MDA and ROS levels $(p<0.01)$ in comparison with GEN treated group, while ATO pretreatment wasn't able to decrease GEN-induced elevation of MDA and ROS levels. MT and ATO combination significantly decreased renal MDA and ROS levels $(p<0.001)$ in comparison with GEN treated and ATO pretreated groups (Figure 1-2).

Treatment effects of ATO, MT and a combination of them on renal SOD and CAT activity in GEN-treated rats 
CAT and SOD activity significantly decreased in GEN treated group in comparison with control group $(p<0.001)$. Pretreatment with ATO, MT and a combination of them significantly elevated SOD activity $(p<0.05, p<0.01$ and $p<$ 0.001, respectively; Figure 3) in comparison with GEN treated group. In comparison with GEN treated group, renal CAT activity showed a highly significant elevation with ATO $(p<0.05)$, MT $(p<0.001)$ and a combination of ATO and MT pretreatment ( $p<0.001$; Figure 4).

\section{Effects of ATO, MT and a combination of them on renal GSH level in GEN- treated rats}

GSH level significantly decreased in GEN treated group in comparison with control group $(p<0.001)$. Pretreatment with ATO and MT showed a significant elevation in renal GSH level ( $p<0.05$ and $p<0.01$, respectively) in comparison with GEN treated group. Pretreatment with a combination of ATO and MT significantly elevated renal GSH level in comparison with GEN treated and ATO pretreated groups $(p<0.001$; Figure 5).

\section{Discussion}

The clinical usefulness of GEN, a widely used aminoglycoside antibiotic for the treatment of severe gram-negative bacterial infections, is limited due to its nephrotoxicity (Shakil et al. 2008). Results of our study confirmed that all rats injected with GEN at a dose of $100 \mathrm{mg} / \mathrm{kg}$ (i.p.) for 8 consecutive days, presented a typical pattern of nephrotoxicity which was correlated with elevated levels of serum urea and creatinine levels that this impairment of renal function was accompanied by an increase in kidneys weight, renal ROS and MDA levels and reduction in renal GSH level, SOD and CAT activity. Our data showed the 
pretreatment of rats with MT (20 mg/kg/day, p.o.) for 7 days was able to attenuate GEN-induced nephrotoxicity by preventing from GEN-induced increase in renal ROS, MDA, serum urea and creatinine levels and increasing the activity and level of antioxidative defense. Despite the improvement of antioxidant activity and level, ATO (10 mg/kg/day, p.o.) couldn't reduce renal ROS, MDA, serum urea and creatinine levels but in the presence of ATO, the beneficial effects of MT were maintained or improved.

According to experimental data, oxygen free radicals are considered to be important mediators of GEN-mediated tubular necrosis and the decrease in glomerular filtration rate. Some investigators showed that GEN enhances the generation of reactive oxygen and nitrogen species by releasing iron from renal cortical mitochondria and forming iron-GEN complexes through iron chelation, which is a potent catalyst of free-radical formation and ROS generation (BALIGA et al. 1999; Priuska and Schacht 1995; Yanagida et al. 2004). It has been shown that GEN enhances the production of $\mathrm{O} 2, \mathrm{H} 2 \mathrm{O} 2$ and hydroxyl radicals that can cause changes in some macromolecules via several mechanisms including membrane lipids peroxidation, nitrotyrosine formation, protein oxidation and DNA damage (Cuzzocrea et al. 2002; Fauconneau et al. 1995). Increased production of ROS is supported by elevation of serum urea and creatinine levels, MDA level, an index of LPO, depletion of kidney reduced GSH content and decrease in antioxidant enzymes activity which could aggravate the oxidative damage (Vijayalekshmy et al. 1992; Yazar et al. 2003).

The results of our study showed that pretreatment with MT, induced a significant decrease in kidneys weight, serum urea and creatinine levels and efficiently improved the GEN-induced elevation of ROS and MDA levels and suppression of GSH level, SOD and CAT activity in kidney tissue. In mammals, MT1 and MT2 are MT receptor system. These receptors are diversely distributed in various tissues 
and organs. The MT1 subtype is found in kidneys, brain, and retina, particularly in the SCNs that could be a reason to regulatory effects of MT on circadian rhythm, reproduction and peripheral vasoconstriction. The MT2 subtype is found in retina and brain but not in SCNs. It has been suggested that MT2 subtype has a role in the regulation of body temperature. Besides membrane receptor signaling, MT has receptor-independent effects by interacting with and freely crossing the cell membrane, in which it can interact with intracellular enzymes, transporters, cytoskeletal proteins and nuclear receptors. Numerous studies have shown that receptor-independent functions of MT may depend on its antioxidant activity (Luchetti et al. 2010). MT shows antioxidant effect directly by scavenging free radicals or indirectly by increasing the activity and expression of antioxidative defense (Allegra et al. 2003; Rodriguez et al. 2004). MT is a very potent scavenger of ROS including singlet oxygen $\left({ }^{1} \mathrm{O}_{2}\right)$, superoxide radical $\left(\mathrm{O}_{2}{ }^{-}\right)$, hydroxyl radical $(\mathrm{OH})$ and hydrogen peroxide $\left(\mathrm{H}_{2} \mathrm{O}_{2}\right)$. Also MT scavenges nitric oxide (NO) and peroxynitrite anion (ONOO-, formed when NO couples with O2) and decreases their production by inhibiting nitric oxide synthase activity and down-regulating the expression of inducible nitric oxide synthase (iNOS) (Karbownik et al. 2001). In addition, MT increases intracellular GSH concentration by stimulation of its rate limiting enzyme, $\gamma$-glutamylcysteine synthase and stimulates the activity or gene expression of endogenous antioxidant enzymes; i.e. SOD (dismutases $\mathrm{O}_{2}{ }^{--}$to $\mathrm{H}_{2} \mathrm{O}_{2}$ ), CAT (converts $\mathrm{H}_{2} \mathrm{O}_{2}$ to water and oxygen), GPX (oxidizes $\mathrm{GSH}$ to Glutathione disulfide (GSSG)) and GRd (reduces GSSG to GSH) by interacting with either membrane or nuclear receptors. It has been shown that there are mitochondrial binding sites for MT by which can elevate mitochondrial ATP production- that is vital for all cellular functions such as repair of oxidatively damaged molecules- during mitochondrial treatment with it. It was reported that MT increases the efficiency of mitochondrial oxidative phosphorylation metabolic 
pathway and inhibits NADPH-dependent lipid peroxidation in mitochondria. Additionally, studies show that the number of cell mitochondria increases following long term MT administration (Absi et al. 2000; Acuña-Castroviejo et al. 2001; Martin et al. 2000; Reiter et al. 2003). MT has been shown to ameliorate early glomerulopathy mediated by oxidative stress in diabetic rats' kidneys (Ha et al. 1999). MT has been found to be protective against renal oxidative damage induced by aluminium, adriamycin, lead, ochratoxin and Mechlorethamine (Kunak et al. 2012; Mahieu et al. 2009; Martínez-Alfaro et al. 2013; Meki and Hussein 2001; Montilla et al. 1997; Ramirez-Garcia et al. 2015). It has been shown that MT is able to reduce renal failure mediated by glycerol, spinal cord and thermal injury. It can also prevent cyclosporine ROS-induced renal functional impairment in rats without alteration in cyclosporine plasma levels (Akakin et al. 2013; Malhotra et al. 2004; Mun and Suh 2000; Şener et al. 2002). The MT treatment has been demonstrated as a protection against GEN-induced nephrotoxicity that our results are entirely consistent with theirs (Lee et al. 2012; Özbek et al. 2000; Sener et al. 2002; Shifow et al. 2000). Our results also showed that pretreatment with ATO alone wasn't significantly able to decrease kidneys weight, serum urea and creatinine levels and elevated levels of ROS and MDA induced by GEN in kidney tissue but it efficiently improved suppression of GSH level as well as SOD and CAT activity. ATO is a member of statins family that is widely used for the reduction in cholesterol serum levels by competitively inhibiting the enzyme HMG-CoA (3-hydroxy-3-methyl-glutaryl-coenzyme A) reductase, thus reducing the availability of mevalonate and cholesterol biosynthesis (Kapur and Musunuru 2008). In addition to its anti-hypercholesterolemic effects, the positive clinical efficacy of ATO may derive from its ability to inhibit oxidative stress by interfering with specific oxidant species-generating pathways. ATO has been shown to reduce serum level of MDA, ox-LDL and several markers of oxidative 
stress, such as nitrotyrosine, 8-hydroxydeoxyguanosine (8-OHdG) and isoprostanes, and increase antioxidants such as SOD and CAT. It has been suggested that ATO can exert cellular antioxidant effects by multiple mechanisms including down-regulation of circulating levels of NADPH oxidase, one of the most important cellular sources of superoxide anion production. The downregulation of NADPH oxidase by ATO is postulated to occur via its direct inhibitory effect on NADPH oxidase or the up-regulation of adiponectin (APN) production that leads to the inhibition of NADPH oxidase (Cangemi et al. 2007; Roberto et al. 2010). The protective effect of ATO against GEN -induced nephrotoxicity has been demonstrated and it is found that ATO prevents the toxic effects of GEN in kidney via the inhibition of MAPK and NF-kB signaling pathways and iNOS expression by antioxidant activity (Ozbek et al. 2009). Also our data shows that pretreatment with a combination of MT plus ATO can enhance the beneficial effects of ATO against GEN-induced nephrotoxicity as serum urea, kidney ROS and MDA levels significantly reduced in comparison while in ATO group alone and in the presence of ATO, the beneficial effects of MT didn't change or even improved. In addition the $p$ value in ATO plus MT group was lesser than MT group which suggested that the effects of ATO plus MT group are not only related to MT. The combination therapy of ATO and MT has been evaluated in some studies. It has been shown that joining treatment with ATO and MT can improve histopathological changes induced by oxidative damages in pancreas of diabetic rats (Gurpinar et al. 2010). Another study indicates that MT can improve ATO beneficial effects and reduce its side effects on endothelial cell damage induced by inflammation and oxidative stress injury mediated by bacterial lipopolysaccharide (LPS) that our results are entirely consistent with their results (Dayoub et al. 2011). 


\section{Conclusions}

From the results of the present study, it could be inferred that the pretreatment of rats with MT and ATO inhibits GEN-induced nephrotoxicity and it seems that MT can enhance the beneficial effects of ATO against GEN-induced nephrotoxicity. Administration of MT plus ATO prior to GEN exposure resulted in a marked decline in kidneys weight, serum urea and creatinine concentration, renal ROS and MDA levels and also significant elevation in renal GSH level, SOD and CAT activity. These results suggest the protective effect of ATO and MT may be caused by their antioxidant properties.

\section{Conflict of interest}

There is no conflict of interest to declare.

\section{References}

Abdel-Raheem, I.T., Abdel-Ghany, A.A., and Mohamed, G.A. 2009. Protective effect of quercetin against gentamicin-induced nephrotoxicity in rats. Biological and Pharmaceutical Bulletin, 32(1): 61-67. doi:10.1248/bpb.32.61. PMID:19122282.

Absi, E., Ayala, A., Machado, A., and Parrado, J. 2000. Protective effect of melatonin against the 1-methyl-4-phenylpyridinium-induced inhibition of Complex I of the mitochondrial respiratory chain. J. Pineal Res. 29(1): 40-47. doi:10.1034/j.1600-079X.2000.290106.x. PMID:10949539.

Acuña-Castroviejo, D., Martín, M., Macías, M., Escames, G., León, J., Khaldy, H., and Reiter, R.J. 2001. Melatonin, mitochondria, and cellular bioenergetics. J. Pineal Res. 30(2): 65-74. doi:10.1034/j.1600-079X.2001.300201.x. PMID:11270481.

Akakin, D., Kiran, D., Ozkan, N., Erşahin, M., Ozdemir-Kumral, Z.N., Yeğen, B., and Şener, G. 2013. Protective effects of melatonin against spinal cord injury induced oxidative damage in rat kidney: A morphological and biochemical study. 
Acta Histochemica, 115(8): 827-834. doi:10.1016/j.acthis.2013.04.005. PMID:23725902.

Allegra, M., Reiter, R., Tan, D.X., Gentile, C., Tesoriere, L., and Livrea, M. 2003. The chemistry of melatonin's interaction with reactive species. J. Pineal Res. 34(1): 1-10. doi:10.1034/j.1600-079X.2003.02112.x. PMID:12485365.

Balakumar, P., Rohilla, A., and Thangathirupathi, A. 2010. Gentamicin-induced nephrotoxicity: do we have a promising therapeutic approach to blunt it? Pharmacological Research, 62(3): 179-186. doi:10.1016/j.phrs.2010.04.004. PMID:20434560.

Baliga R, Ueda N, Walker PD, Shah SV.1999. Oxidant mechanisms in toxic acute renal failure. Drug Metab. Rev. 31(4): 971-997. doi:10.1081/DMR-100101947. PMID:10575556.

Begg, E.J., and Barclay, M.L. 1995. Aminoglycosides--50 years on. Br. J. Clin. Pharmacol. 39(6): 597-603. PMID:7654476.

Cangemi, R., Loffredo, L., Carnevale, R., Perri, L., Patrizi, M.P., Sanguigni, V., Pignatelli, P., and Violi, F. 2007. Early decrease of oxidative stress by atorvastatin in hypercholesterolaemic patients: effect on circulating vitamin E. Eur. Heart J. 29(1): 54-62. doi:10.1093/eurheartj/ehm565. PMID:18065424.

Cuzzocrea, S., Mazzon, E., Dugo, L., Serraino, I., Di Paola, R., Britti, D., De Sarro, A., Pierpaoli, S., Caputi, A.P., and Masini, E. 2002. A role for superoxide in gentamicin-mediated nephropathy in rats. Eur. J. Pharmacol. 450(1): 67-76. doi:10.1016/S0014-2999(02)01749-1. PMID:12176111.

Dam, V.P., Scott, J.L., Ross, A., and Kinobe, R.T. 2012. Inhibition of cystathionine gamma-lyase and the biosynthesis of endogenous hydrogen sulphide ameliorates gentamicin-induced nephrotoxicity. Eur. J. Pharmacol. 685(1): 165173. doi:10.1016/j.ejphar.2012.04.030. PMID:22546230.

Davignon, J., Jacob, R.F., and Mason, R.P. 2004. The antioxidant effects of statins. Coronary Artery Disease, 15(5): 251-258. PMID: 15238821.

Dayoub, J.C., Ortiz, F., López, L.C., Venegas, C., del Pino-Zumaquero, A., Roda, O., Sánchez-Montesinos, I., Acuña-Castroviejo, D., and Escames, G. 2011. Synergism between melatonin and atorvastatin against endothelial cell damage induced by lipopolysaccharide. J. Pineal Res. 51(3): 324-330. doi:10.1111/j.1600079X.2011.00892.x. PMID:21585521. 
Fauconneau, B., Tallineau, C., Huguet, F., and Piriou, A. 1995. Gentamicininduced kidney damage and lipid peroxidation in rats. Toxicol. Lett. 76(2): 127134. doi:10.1016/0378-4274(94)03205-L. PMID:7725344.

Arendt, J. 1995. Melatonin and the mammalian pineal gland, London: Chapman Hall.

Greenwood, J., Steinman, L., and Zamvil, S.S. 2006. Statin therapy and autoimmune disease: from protein prenylation to immunomodulation. Nature Reviews Immunology, 6(5): 358-370. doi:10.1038/nri1839. PMID:16639429.

Gurpinar, T., Ekerbicer, N., Uysal, N., Barut, T., Tarakci, F., and Tuglu, M.I. 2010. The histologic evaluation of atorvastatin and melatonin treatment on oxidative stress and apoptosis of diabetic rat pancreas. Kafkas Univ Vet Fak Derg 16(4): 547-552.

Ha, H., Yu, M.-R., and Kim, K.H. 1999. Melatonin and taurine reduce early glomerulopathy in diabetic rats. Free Radic. Biol. Med. 26(7): 944-950. doi:10.1016/S0891-5849(98)00276-7. PMID:10232838.

Kapur, N.K., and Musunuru, K. 2008. Clinical efficacy and safety of statins in managing cardiovascular risk. Vascular health and risk management, 4(2): 341. doi:10.2147/VHRM.S1653. PMID:18561510.

Karbownik, M., Reiter, R.J., Cabrera, J., and Garcia, J.J. 2001. Comparison of the protective effect of melatonin with other antioxidants in the hamster kidney model of estradiol-induced DNA damage. Mutation Research/Fundamental and Molecular Mechanisms of Mutagenesis, 474(1): 87-92. doi:10.1016/S00275107(00)00164-0. PMID:11239965.

Kunak, Z.I., Macit, E., Yaren, H., Yaman, H., Cakir, E., Aydin, I., Turker, T., Kurt, Y.G., Ozcan, A., and Uysal, B. 2012. Protective effects of melatonin and smethylisothiourea on mechlorethamine induced nephrotoxicity. Journal of Surgical Research, 175(1): e17-e23. doi:10.1016/j.jss.2011.11.002. PMID:22261582.

Kuo, C.-H., Maita, K., Sleight, S.D., and Hook, J.B. 1983. Lipid peroxidation: a possible mechanism of cephaloridine-induced nephrotoxicity. Toxicology and applied pharmacology, 67(1): 78-88. PMID:6845359.

Kwak, B., Mulhaupt, F., Myit, S., and Mach, F. 2000. Statins as a newly recognized type of immunomodulator. Nature medicine, 6(12): 1399-1402. 10.1038/82219. PMID:11100127. 
Lee, I.-C., Kim, S.-H., Lee, S.-M., Baek, H.-S., Moon, C., Kim, S.-H., Park, S.-C., Kim, H.-C., and Kim, J.-C. 2012. Melatonin attenuates gentamicin-induced nephrotoxicity and oxidative stress in rats. Arch. Toxicol. 86(10): 1527-1536. doi:10.1007/s00204-012-0849-8. PMID:22526374.

Lopez-Novoa, J.M., Quiros, Y., Vicente, L., Morales, A.I., and Lopez-Hernandez, F.J. 2011. New insights into the mechanism of aminoglycoside nephrotoxicity: an integrative point of view. Kidney international, 79(1): 3345. doi:10.1038/ki.2010.337. PMID:20861826.

Luchetti, F., Canonico, B., Betti, M., Arcangeletti, M., Pilolli, F., Piroddi, M., Canesi, L., Papa, S., and Galli, F. 2010. Melatonin signaling and cell protection function. FASEB J. 24(10): 3603-3624. doi:10.1096/fj.10-154450. PMID:20534884.

Mahieu, S., del Carmen Contini, M., González, M., and Millen, N. 2009. Melatonin reduces oxidative damage induced by aluminium in rat kidney. Toxicol. Lett. 190(1): 9-15. doi:10.1016/j.toxlet.2009.06.852. PMID:19539013.

Malhotra, S., Sawhney, G., and Pandhi, P. 2004. The therapeutic potential of melatonin: a review of the science. Medscape General Medicine, 6(2). PMID:15266271.

Martin, M., Macias, M., Escames, G., Reiter, R., Agapito, M., Ortiz, G., and Acuna-Castroviejo, D. 2000. Melatonin-induced increased activity of the respiratory chain complexes I and IV can prevent mitochondrial damage induced by ruthenium red in vivo. J. Pineal Res. 28(4): 242-248. doi:10.1034/j.1600 079X.2000.280407.x. PMID:10831160.

Martínez-Alfaro, M., Ramírez-García, G., Gutiérrez-Granados, S., AlcarazContreras, Y., Gallegos-Corona, M.-A., de Larrea, G.Z.L., and Cárabez-Trejo, A. 2013. Melatonin attenuates the effects of sub-acute administration of lead on kidneys in rats without altering the lead-induced reduction in nitric oxide. Journal of Trace Elements in Medicine and Biology, 27(4): 364-369. doi:10.1016/j.jtemb.2013.05.003. PMID:23992869.

Mathew, T.H. 1992. Drug-induced renal disease. The Medical journal of Australia, 156(10): 724-728. PMID:1620020.

Meki, A.-R.M., and Hussein, A.A. 2001. Melatonin reduces oxidative stress induced by ochratoxin $\mathrm{A}$ in rat liver and kidney. Comparative Biochemistry and Physiology C, 130(3): 305-313. PMID:11701387. 
Montilla, P., Tunez, I., Munoz, M., Lopez, A., and Soria, J. 1997. Hyperlipidemic nephropathy induced by adriamycin: effect of melatonin administration. Nephron, 76(3): 345-350. PMID:9226237.

Mun, K.C., and Suh, S.I. 2000. Effect of melatonin on renal function in cyclosporine nephrotoxicity. Transplantation Proc. 32(7): 1919-1920. doi:10.1016/S0041-1345(00)01492-5. PMID:11120000.

Ohkawa, H., Ohishi, N., and Yagi, K. 1979. Assay for lipid peroxides in animal tissues by thiobarbituric acid reaction. Anal. Biochem. 95(2): 351-358. PMID:36810.

Ozbek, E., Cekmen, M., Ilbey, Y.O., Simsek, A., Polat, E.C., and Somay, A. 2009. Atorvastatin prevents gentamicin-induced renal damage in rats through the inhibition of p38-MAPK and NF-kB pathways. Renal failure, 31(5): 382-392. PMID:19839839.

Özbek, E., Turkoz, Y., Sahna, E., Ozugurlu, F., Mizrak, B., and Ozbek, M. 2000. Melatonin administration prevents the nephrotoxicity induced by gentamicin. BJU international, 85(6): 742-746. doi:10.1046/j.1464-410x.2000.00531.x. PMID:10759677

Ozler, M., Korkmaz, A., Uysal, B., Simsek, K., Ozkan, C., Topal, T., and Oter, S. 2011. Effects of topical melatonin and vitamin $\mathrm{E}$ in a rat ischemic wound model. J. Exp. Integ. Med. 1(2): 123-129. doi: 10.5455/jeim.080411.or.006.

Pignatelli, P., Carnevale, R., Cangemi, R., Loffredo, L., Sanguigni, V., Stefanutti, C., Basili, S., and Violi, F. 2010. Atorvastatin inhibits gp91phox circulating levels in patients with hypercholesterolemia. Arteriosclerosis, Thrombosis, and Vascular Biology, 30(2): 360-367. doi:10.1161/ATVBAHA.109.198622. PMID:19965781

Priuska, E.M., and Schacht, J. 1995. Formation of free radicals by gentamicin and iron and evidence for an iron/gentamicin complex. Biochem. Pharmacol. 50(11): 1749-1752. doi:10.1016/0006-2952(95)02160-4. PMID:8615852.

Ramirez-Garcia, G., Martinez-Alfaro, M., Gutierrez-Granados, S., Alatorre-Ordaz, A., Griveau, S., and Bedioui, F. 2015. Electrochemical Assessment of Possible Melatonin Effect On Nitric Oxide Production From Kidneys Of Sub-Acute Lead Treated Rats. Electrochimica Acta, 166: 88-92. doi:10.1016/j.electacta.2015.03.063. 
Reiter, R.J. 1991. Cell biology of its synthesis and of its physiological interactions. Endocrine Rev. 12(2): 151-180. doi:10.1210/edrv-12-2-151 PMID:1649044.

Reiter, R.J., Calvo, J.R., Karbownik, M., Qi, W., and Tan, D.X. 2000. Melatonin and its relation to the immune system and inflammation. Ann. N.Y. Acad. Sci. 917(1): 376-386. doi:10.1111/j.1749-6632.2000.tb05402.x. PMID:11268363

Reiter, R.J., Tan, D.-x., Mayo, J.C., Sainz, R.M., Leon, J., and Czarnocki, Z. 2003. Melatonin as an antioxidant: biochemical mechanisms and pathophysiological implications in humans. Acta Biochim Pol. 50(4): 1129-1146. PMID:14740000.

Reiter, R.J., Tan, D.X., and Maldonado, M.D. 2005. Melatonin as an antioxidant: physiology versus pharmacology. J. Pineal Res. 39(2): 215-216. doi:10.1111/j.1600-079X.2005.00261.x. PMID:16098101.

Carnevale, R., Pignatelli, P., Di Santo, S., Bartimoccia, S., Sanguigni, V., Napoleone, L., Tanzilli, G., Basili, S., Violi, F. 2010. Atorvastatin inhibits oxidative stress via adiponectin-mediated NADPH oxidase down-regulation in hypercholesterolemic patients. Atherosclerosis, 213(1): 225-234. doi:10.1016/j.atherosclerosis.2010.08.056. PMID:20832062.

Rodriguez, C., Mayo, J.C., Sainz, R.M., Antolin, I., Herrera, F., Martin, V., and Reiter, R.J. 2004. Regulation of antioxidant enzymes: a significant role for melatonin. J. Pineal Res. 36(1): 1-9. doi:10.1046/j.1600-079X.2003.00092.x. PMID:14675124.

Romero, F., Pérez, M., Chávez, M., Parra, G., and Durante, P. 2009. Effect of Uric Acid on Gentamicin-Induced Nephrotoxicity in Rats-Role of Matrix Metalloproteinases 2 and 9. Basic Clin. Pharmacol. Toxicol. 105(6): 416-424. doi:10.1111/j.1742-7843.2009.00466.x. PMID:19821833.

Sehajpal, J., Kaur, T., Bhatti, R., and Singh, A.P. 2014. Role of progesterone in melatonin-mediated protection against acute kidney injury. Journal of Surgical Research, 191(2): 441-447. doi:10.1016/j.jss.2014.04.025. PMID:24878191.

Sener, G., Sehirli, A.Ö., Altunbas, H.Z., Ersoy, Y., Paskaloglu, K., Arbak, S., and Ayanoglu-Dulger, G. 2002. Melatonin protects against gentamicin-induced nephrotoxicity in rats. J. Pineal Res. 32(4): 231-236. doi:10.1034/j.1600079X.2002.01858.x. PMID:11982792.

Şener, G., Şehirli, A.Ö., Şatıroğlu, H., Keyer-Uysal, M., and Yeğen, B.Ç. 2002. Melatonin prevents oxidative kidney damage in a rat model of thermal injury. Life 
Sciences, 70(25): 2977-2985. doi:10.1016/S0024-3205(02)01571-0. PMID:12138011.

Shakil, S., Khan, R., Zarrilli, R., and Khan, A.U. 2008. Aminoglycosides versus bacteria-a description of the action, resistance mechanism, and nosocomial battleground. J. Biomed. Sci. 15(1): 5-14. PMID:17657587.

Shifow, A., Kumar, K., Naidu, M., and Ratnakar, K. 2000. Melatonin, a pineal hormone with antioxidant property, protects against gentamicin-induced nephrotoxicity in rats. Nephron, 85(2): 167-174. PMID:10867523.

Shinomol, G.K., and Muralidhara. 2007. Differential induction of oxidative impairments in brain regions of male mice following subchronic consumption of Khesari dhal (Lathyrus sativus) and detoxified Khesari dhal. Neurotoxicology, 28(4): 798-806. doi:10.1016/j.neuro.2007.03.002. PMID:17451808.

Shishehbor, M.H., Brennan, M.-L., Aviles, R.J., Fu, X., Penn, M.S., Sprecher, D.L., and Hazen, S.L. 2003. Statins promote potent systemic antioxidant effects through specific inflammatory pathways. Circulation, 108(4): 426-431. doi:10.1161/01.CIR.0000080895.05158.8B. PMID:12860913.

Soliman, K.M., Abdul-Hamid, M., and Othman, A.I. 2007. Effect of carnosine on gentamicin-induced nephrotoxicity. Medical Science and Technology, 13(3): BR73-BR83. PMID:17325631.

Vijayalekshmy, K., Menon, V., and Leelamma, S. 1992. Role of antibiotics in lipid peroxidation. Ind. J. Biochem. Biophys. 29(4): 371-374. PMID:1427966.

Wassmann, S., Laufs, U., Müller, K., Konkol, C., Ahlbory, K., Bäumer, A.T., Linz, W., Böhm, M., and Nickenig, G. 2002. Cellular antioxidant effects of atorvastatin in vitro and in vivo. Arteriosclerosis, Thrombosis, and Vascular Biology, 22(2): 300-305. PMID:11834532.

Yanagida, C., Ito, K., Komiya, I., and Horie, T. 2004. Protective effect of fosfomycin on gentamicin-induced lipid peroxidation of rat renal tissue. Chemicobiological interactions, 148(3): 139-147. doi:10.1016/j.cbi.2004.05.005. PMID:15276870.

Yazar, E., Elmas, M., Altunok, V., Sivrikaya, A., Oztekin, E., and Birdane, Y.O. 2003. Effects of aminoglycoside antibiotics on renal antioxidants, malondialdehyde levels, and some serum biochemical parameters. Can. J. Vet. Res. 67(3): 239. PMID:12889734. 
Yoshida, M. 2003. Potential role of statins in inflammation and atherosclerosis. Journal of Atherosclerosis and Thrombosis, 10(3): 140-144. doi:10.5551/jat.10.140. PMID:14564082. 
Table 1. Effects of MT and ATO on GEN-induced alterations in the Body weight changes and kidney weights (The data were expressed as mean \pm S.E.M., $n=6$ ).

\begin{tabular}{|c|c|c|c|c|c|}
\hline \multirow[t]{2}{*}{ Items } & \multicolumn{3}{|l|}{ Group } & \multirow[b]{2}{*}{ GEN + MT } & \multirow[b]{2}{*}{ GEN + ATO + MT } \\
\hline & Control & GEN & GEN + ATO & & \\
\hline Body weight (g) & $369.2 \pm 8.06$ & $349.2 \pm 4.91$ & $354.7 \pm 6.79$ & $360.2 \pm 2.72$ & $363.5 \pm 2.67$ \\
\hline Kidney (g) & $0.807 \pm 0.038$ & $0.958 \pm 0.021^{*}$ & $0.867 \pm 0.015$ & $0.831 \pm 0.011^{\#}$ & $0.835 \pm 0.028^{\#}$ \\
\hline $\begin{array}{c}\text { Per body weight } \\
(\%)\end{array}$ & $0.222 \pm 0.012$ & $0.275 \pm 0.008^{* *}$ & $0.245 \pm 0.008$ & $0.231 \pm 0.004^{\#}$ & $0.234 \pm 0.009^{\#}$ \\
\hline
\end{tabular}

The data analyzed by one-way analysis of variance (ANOVA) followed by tukey test.

${ }^{*} p<0.05,{ }^{* *} p<0.01$ compared to the control group.

${ }^{\#} p<0.05$ compared to the GEN group.

Table 2. Effects of MT and ATO on GEN-induced alterations in the serum biochemical parameters (The data were expressed as mean \pm S.E.M., $n=6$ ).

$\begin{array}{ccc}\text { Group } & \text { Creatinine }(\mathbf{m g} / \mathbf{d l}) & \text { BUN }(\mathbf{m g} / \mathbf{d L}) \\ \text { Control } & 0.71 \pm 0.06 & 23.4 \pm 1.1 \\ \text { GEN } & 0.95 \pm 0.02^{* *} & 42.24 \pm 3.23^{* * *} \\ \text { GEN + ATO } & 0.86 \pm 0.03 & 34.88 \pm 2.65^{* *} \\ \text { GEN + MT } & 0.75 \pm 0.02^{\#} & 29.15 \pm 0.97^{\# \#} \\ \text { GEN + ATO + MT } & 0.72 \pm 0.03^{\# \#} & 26.87 \pm 1.13^{\# \#, \dagger}\end{array}$

The data analyzed by one-way analysis of variance (ANOVA) followed by tukey test.

${ }^{* *} p<0.01,{ }^{* * *} p<0.001$ compared to the control group; ${ }^{\#} p<0.05,{ }^{\# \#} p<0.01,{ }^{\# \# \#} p<0.001$ compared to the GEN group; ${ }^{\dagger} p<0.05$ compared to the GEN + ATO group. 


\section{Legends}

Fig. 1. Levels of lipid peroxidation in the kidneys of control and experimental animals (The data were expressed as mean \pm S.E.M., $n=6$ ). ${ }^{* * *} p<0.01, \quad{ }^{* * * *} p<0.001$ compared to the control group; ${ }^{\# \#} p<0.01,{ }^{\# \#}$ $p<0.001$ compared to the GEN group; ${ }^{\dagger \dagger \dagger} p<0.001$ compared to the GEN + ATO group.

Fig. 2. Levels of ROS (\% of control) in the kidneys of control and experimental animals (The data were expressed as mean \pm S.E.M., $n=6) .{ }^{* *} p<0.01,{ }^{* * *} \mathrm{p}<0.001$ compared to the control group; ${ }^{\# \#} p<0.01,{ }^{\# \#}$ $p<0.001$ compared to the GEN group; ${ }^{\dagger \dagger \dagger} p<0.001$ compared to the GEN + ATO group.

Fig. 3. Levels of SOD in the kidneys of control and experimental animals (The data were expressed as mean \pm S.E.M., $n=6$ ). ${ }^{* * *} p<0.001$ compared to the control group; ${ }^{\# \#} p<0.01,{ }^{\# \#} p<0.001$ compared to the GEN group; ${ }^{\dagger \dagger \dagger} p<0.001$ compared to the GEN + ATO group.

Fig. 4. Levels of Catalase in the kidneys of control and experimental animals (The data were expressed as mean \pm S.E.M., $n=6) .{ }^{* *} p<0.05,{ }^{* * *} p<0.001$ compared to the control group; ${ }^{\#} p<0.05,{ }^{\# \#} p<0.001$ compared to the GEN group; ${ }^{\dagger \dagger} p<0.001$ compared to the GEN + ATO group.

Fig. 5. Levels of GSH in the kidneys of control and experimental animals (The data were expressed as mean \pm S.E.M., $\mathrm{n}=6$ ). ${ }^{* * * *} p<0.001$ compared to the control group; ${ }^{\#} p<0.05,{ }^{\# \#} p<0.01,{ }^{\# \#} p<0.001$ compared to the GENgroup; ${ }^{\dagger \dagger} p<0.001$ compared to the GEN + ATO group. 
Fig. 1.

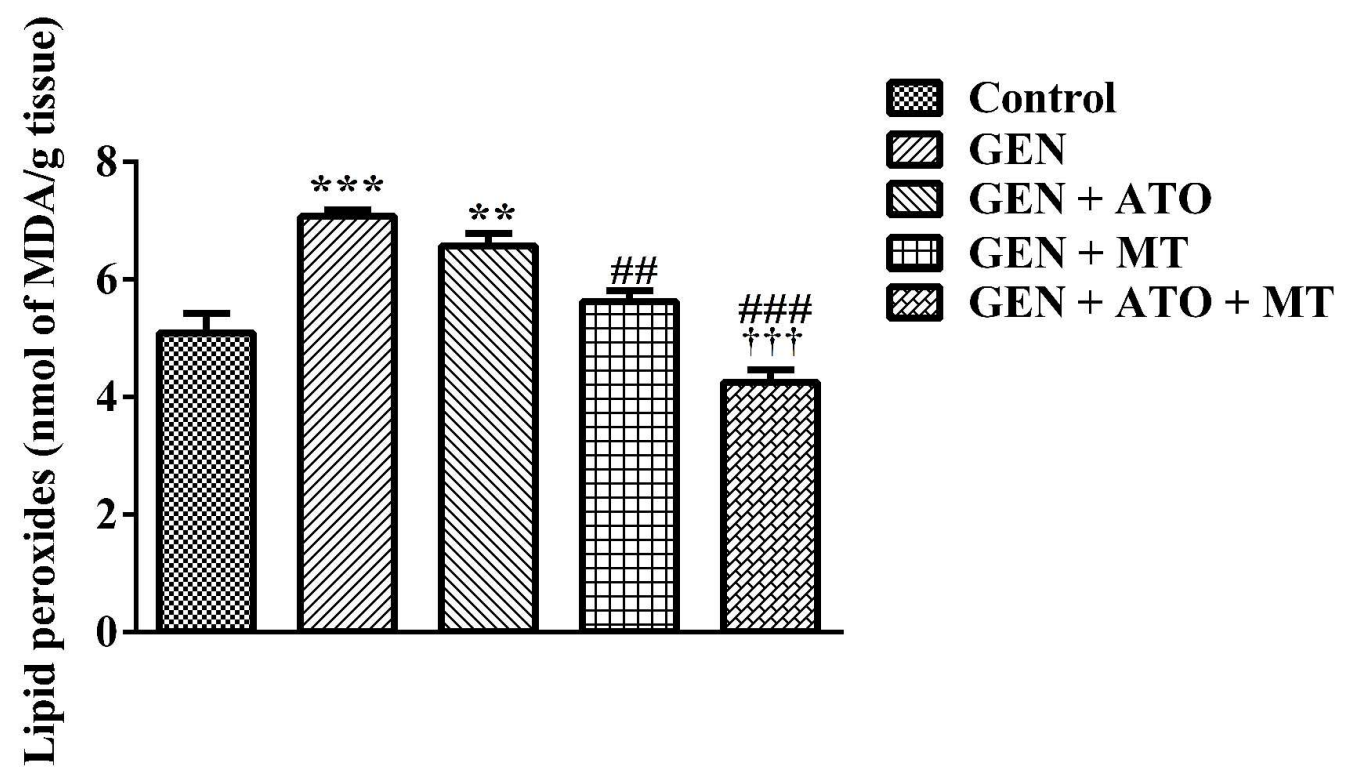

Fig. 2.

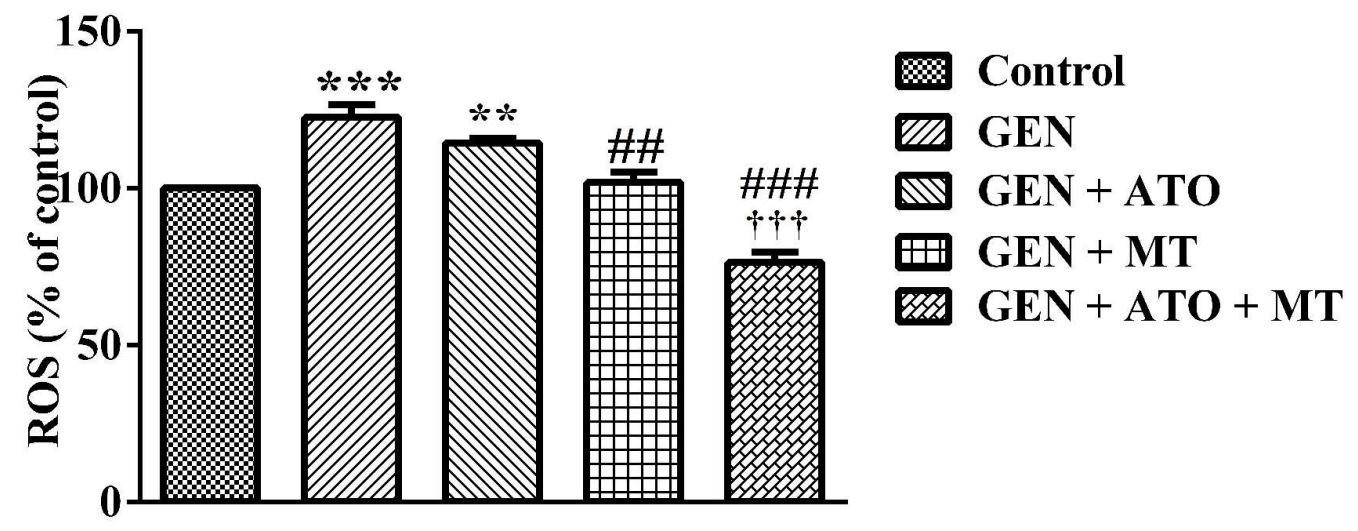


Fig. 3.

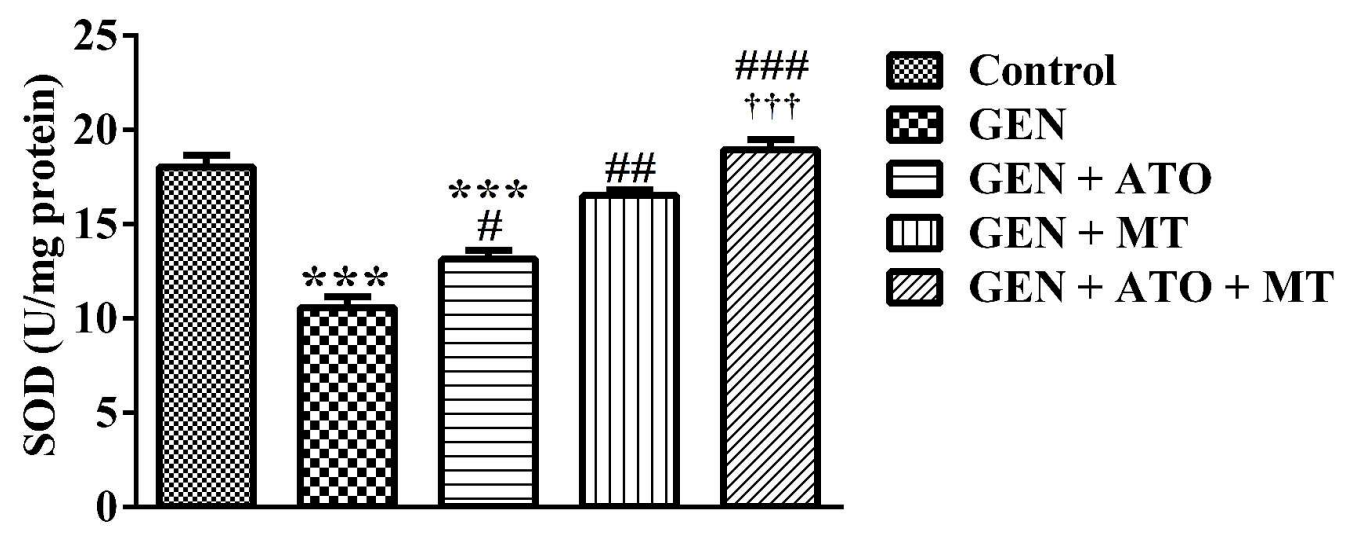

Fig. 4.

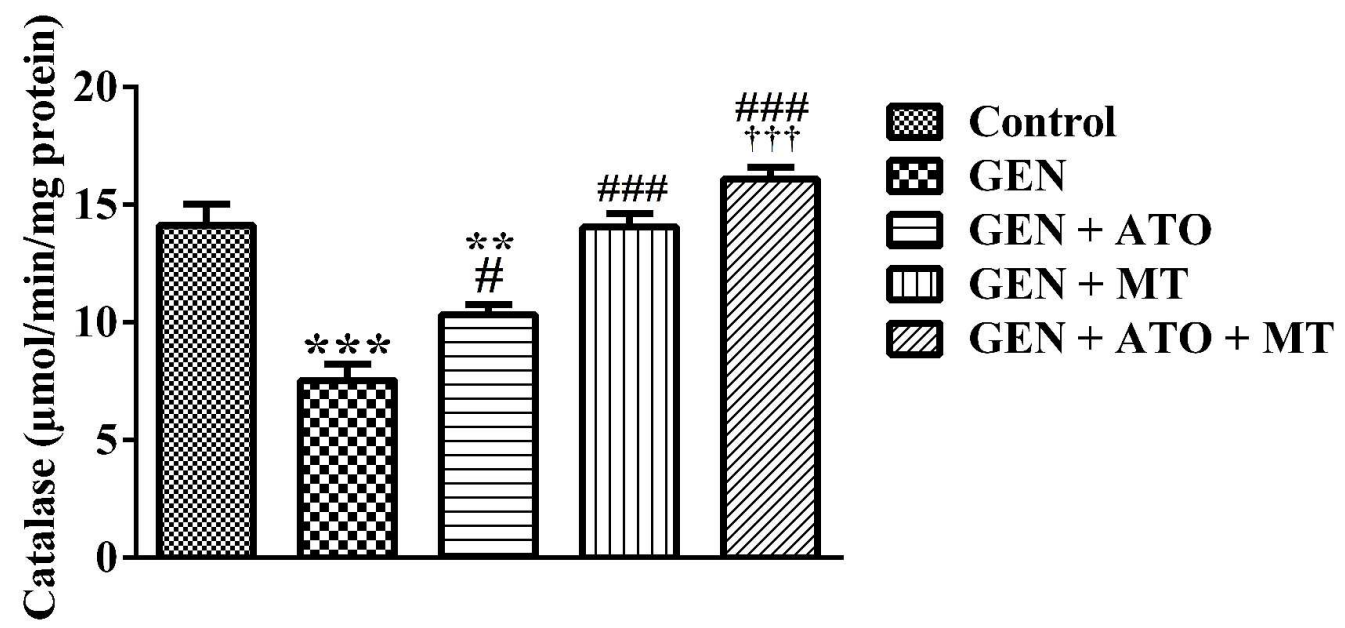


Fig. 5.

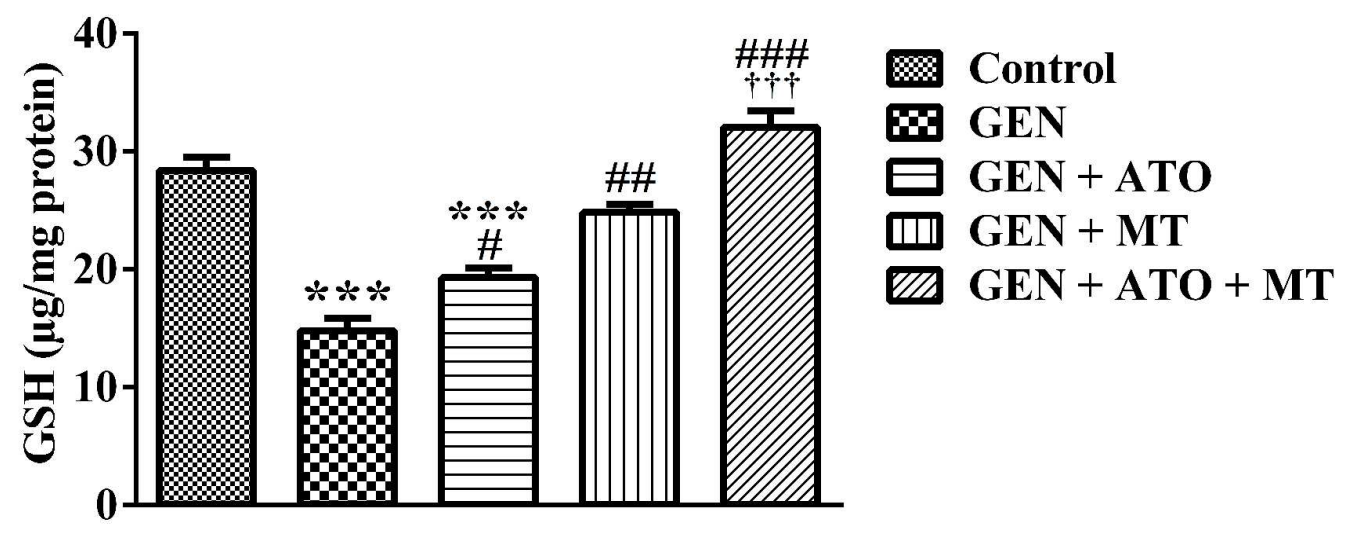

